\title{
Annotation \\ Physical Properties of Pit and Fissure Sealants
}

\author{
J. B. DENNISON and J. M. POWERS
}

The University of Michigan, School of Dentistry, Ann Arbor, Michigan 48109

J Dent Res 58(4): 1430, April 1979

All products currently marketed for the sealing of occlusal pits and fissures utilize a dimethacrylate resin with only minor differences in formulation. Four commercial sealants and a composite restorative resin (V) were evaluated for the physical properties shown in Tables 1 and 2 according to American Dental Association Specification No. 27 for Direct Filling Resins ( $J A D A$ 94:1191, 1977) and Dennison and Craig (JADA $85: 101,1972)$. An unfilled direct resin (S) was also included for comparison.

The sealant (K) filled with $40 \%$ (by weight) quartz had a higher elastic modulus and lower water sorption than the other sealants, but generally behaved more like an unfilled than a composite resin. The lack of improvement of $K$ in resistance to indentation correlated well with previous results on abrasion resistance (Roberts, et al., $J$ Dent Res 56:692, 1977). The sealant (NS) accelerated by ultraviolet light was higher in tensile strength and elastic modulus and lower in water sorption than the other unfilled sealants, possibly indicating a more complete polymerization. The

Received for publication June 19, 1978.

Accepted for publication July 17, 1978 .

This investigation was supported entirely by Biomedical Research Support Grant RR-05321 from the National Institutes of Health, Bethesda, MD 20014.

The cooperation of the following companies in providing commercial products is acknowledged: L. D. Caulk Co., Johnson \& Johnson, Kerr Mfg. Co., and $3 \mathrm{M}$ Co. sealant (D) with an accelerated working time exhibited a slightly harder surface with a greater recovery after indentation. The pigmented sealant $(W)$ had an initial compressive strength similar to the composite restorative material, but displayed greater sensitivity to head speed during compression. All materials behaved viscoelastically.

Although there were some properties in which statistically significant differences were detected, no one sealant showed superiority in all properties. The four sealants generally behaved similarly, more closely approximating properties for the unfilled direct resin than the composite restorative resin. Product selection, therefore, should also take into consideration handling characteristics and the results from forthcoming clinical trials.

TABLE 2

WATER SORPTION OF PIT AND FISSURE SEALANTS*

\begin{tabular}{|c|c|c|}
\hline \multirow[b]{2}{*}{ Material } & \multicolumn{2}{|c|}{ Water Sorption $\left(\mathrm{mg} / \mathrm{cm}^{2}\right)$} \\
\hline & 24 Hrs. & $1 \mathrm{Wk}$ \\
\hline $\mathrm{K}$ & $1.34(0.11)$ & $2.45(0.04)$ \\
\hline NS & $1.80(0.02)$ & $2.88(0.18)$ \\
\hline D & $2.36(0.18)$ & $3.73(0.38)$ \\
\hline $\mathrm{W}$ & $2.22(0.22)$ & $3.51(0.40)$ \\
\hline $\mathrm{V}$ & $0.77(0.31)$ & $1.44(0.17)$ \\
\hline$S$ & $1.58(0.27)$ & $2.03(0.30)$ \\
\hline
\end{tabular}

* Means with standard deviations in parentheses. Tukey intervals $(\alpha=0.05)$ for comparisons among products and between times were 0.39 and 0.27 $\mathrm{mg} / \mathrm{cm}^{2}$, respectively.

TABLE 1

MECHANICAL PROPERTIES OF PIT AND FISSURE SEALANTS*

\begin{tabular}{|c|c|c|c|c|c|c|c|}
\hline \multirow[b]{2}{*}{ Material } & \multicolumn{2}{|c|}{$\begin{array}{c}\text { Compressive Strength (MPa) } \\
\text { Head Speed }\end{array}$} & \multirow{2}{*}{$\begin{array}{l}\text { Tensile } \\
\text { Strength } \\
\text { (MPa) }\end{array}$} & \multirow{2}{*}{$\begin{array}{l}\text { Modulus of } \\
\text { Elasticity } \\
\text { (GPa) }\end{array}$} & \multirow{2}{*}{$\begin{array}{l}\text { Indentation } \\
\text { Depth } \\
(\mu \mathrm{m})\end{array}$} & \multirow{2}{*}{$\begin{array}{l}\text { Recovery } \\
(\%)\end{array}$} & \multirow{2}{*}{$\begin{array}{c}\text { Knoop Hardness } \\
100 \mathrm{gm} . \text { load } \\
\left(\mathrm{kg} / \mathrm{mm}^{2}\right)\end{array}$} \\
\hline & $0.02 \mathrm{~cm} / \mathrm{min}$ & $1.0 \mathrm{~cm} / \mathrm{min}$ & & & & & \\
\hline $\mathrm{Kt}$ & $170(70)$ & $150(21)$ & $30.7(1.8)$ & $5.2 \quad(0.7)$ & $84.7(5.0)$ & $74.6(4.0)$ & $25.4(1.6)$ \\
\hline NS束 & $108(24)$ & $92(18)$ & $33.2(4.6)$ & $2.88(0.35)$ & $88.3(1.5)$ & $83.4(0.4)$ & $15.5(0.8)$ \\
\hline $\mathrm{D} \S$ & $127(45)$ & $92(21)$ & $23.6(4.0)$ & $2.08(0.19)$ & $89.0(1.0)$ & $86.5(1.0)$ & $20.1(1.1)$ \\
\hline$W_{\infty}$ & $230(34)$ & $133(29)$ & $19.8(2.4)$ & $2.60(0.13)$ & $158 \quad(8)$ & $73.5(2.5)$ & $15.6(0.4)$ \\
\hline$V \#$ & $207(32)$ & $225(16)$ & $30.3(6.0)$ & $15.3(1.5)$ & $66.9(0.4)$ & $72.4(0.1)$ & -- \\
\hline SI & $72(2)$ & - & $23.0(1.0)$ & $2.3(0.6)$ & $113.0(3.0)$ & $74.1(3.5)$ & $18.0(0.5)$ \\
\hline \multicolumn{8}{|l|}{ Tukey } \\
\hline \multirow{2}{*}{$\begin{array}{l}\text { Intervals } \\
(\alpha=0.05)\end{array}$} & & & & & & & \\
\hline & $\begin{array}{c}44 \\
\text { among } \\
\text { products }\end{array}$ & $\begin{array}{c}20 \\
\text { between } \\
\text { speeds }\end{array}$ & 6.2 & 0.42 & 1.3 & 6.4 & 0.9 \\
\hline
\end{tabular}

* Means with standard deviations in parentheses

$\dagger$ Kerr Pit and Fissure Sealant, \#0513771083, Kerr Mfg. Co., Romulus, Mich.

$\$$ Nuva Seal, base - 040477, initiator - 120276, L. D. Caulk Co., Milford, Del.

$\S$ Delton, \#10470202, Johnson \& Johnson, East Windsor, N.J.

$\infty$ White Sealant, resin A - 70702, resin B - 7059B1, Minnesota Mining and Mfg. Co., Minneapolis, Minn.

\#Vytol, base - 042077, catalyst - 050477, L. D. Caulk Co., Milford, Del.

I Sevriton, Amalgamated Dental Trade Distribution Ltd., London, Eng.

(Dennison and Craig, $J A D A 85: 101,1972$ and Powers et al., $J$ Dent Res $55: 432,1976$ ) 